01

\title{
Квазиоптическая теория усиления релятивистским электронным потоком поверхностных волн, распространяющихся над гофрированными структурами (импедансное приближение)
}

\author{
(C) Н.С. Гинзбург, А.М. Малкин, И.В. Железнов, А.С. Сергеев, Е.Р. Кочаровская
}

Институт прикладной фризики РАН, 603950 Нижний Новгород, Россия

e-mail: ginzburg@appl.sci-nnov.ru

(Поступило в Редакцию 16 мая 2016 г.)

На основе квазиоптического подхода построена импедансная модель, описывающая усиление монохроматической волны прямолинейно движущимся над гофрированной структурой релятивистским электронным пучком. В рамках данной модели действующая на электроны компонента электрического поля записана с учетом наведенных высокочастотных полей пространственного заряда пучка. В приближении малого сигнала получено дисперсионное уравнение, на основании которого найдены инкременты неустойчивости в различных областях параметров. Эффективность энергообмена на стадии насыщения усиления определена на основе двумерной нелинейной модели, в которой распространение волны описывается параболическим уравнением с излучательным граничным условием. Показана возможность использования исследованной схемы для усиления субмиллиметрового излучения.

\section{Введение}

Стимулированное черенковское излучение релятивистских электронных потоков в периодическигофрированных волноводах широко используется в релятивистской электронике для создания генераторов и усилителей с субгигаваттным и гигаваттным уровнями мощности [1-6]. Теория таких приборов [7,8] хорошо разработана и основана на теории возбуждения волноводов $[9,10]$. Поле излучения представляется в виде объемной волноводной моды, которая, распространяясь в периодической структуре в соответствии с теоремой Флоке, может быть представлена в виде суммы пространственных гармоник. Прямолинейно движущийся электронный пучок взаимодействует с одной из этих гармоник в условиях синхронизма черенковского типа $\omega=(h+n \bar{h}) v$, где $v-$ поступательная скорость частиц, $\omega$ - частота излучения, $h-$ продольное волновое число основной гармоники, $\bar{h}=2 \pi / d, d-$ период структуры, $n$ - номер синхронной пространственной гармоники.

Вместе с тем в последнее время становится актуальной задача освоения коротковолновых, в частности терагерцового, диапазонов частоты, что неизбежно требует использования сверхразмерных электродинамических систем и соответственно квазиоптических методов описания электронно-волнового взаимодействия [11-13]. Для черенковских источников в качестве предельного случая может быть рассмотрена задача о стимулированном излучении в свободном пространстве ленточного электронного пучка, движущегося прямолинейно над периодически-гофрированной металлической поверхностью, вдоль которой могут распространяться медленные поверхностные волны.

В настоящей работе рассматривается усилительный режим черенковского взаимодействия пучка с замедленной основной гармоникой поверхностной волны $(n=0)$ в условиях относительно небольшой глубины гофрировки в масштабе периода структуры и длины волны. Такое приближение вполне адекватно описывает экспериментальную ситуацию в случае излучения релятивистских пучков, когда для организации взаимодействия черенковского типа требуется относительно небольшое замедление волны.

Работа имеет следующую структуру. В разд. 1 в рамках квазиоптического подхода построена импедансная модель, описывающая распространение поверхностных волн над гофрированной структурой в условиях, далеких от брэгговского резонанса. В разд. 2 получена самосогласованная система уравнений, описывающая возбуждение релятивистским электронным пучком поверхностных волн. Запись действующего на электроны поля, включающая продольную компоненту наведенного высокочастотного электронного тока, позволила провести учет полей пространственного заряда пучка. В разд. 3 в приближении малого сигнала получено дисперсионное уравнение, на основании которого найдены продольные инкременты конвективной неустойчивости в различных областях параметров. Анализ нелинейной стадии электронно-волнового взаимодействия, приведенный в разд. 4, позволил определить эффективность энергообмена. Показана возможность использования исследованной схемы для усиления субмиллиметрового излучения.

\section{1. Распространение волн над синусоидально гофрированной поверхностью: импедансное приближение}

Начнем анализ с исследования дисперсионных характеристик поверхностных волн, распространяющихся над 


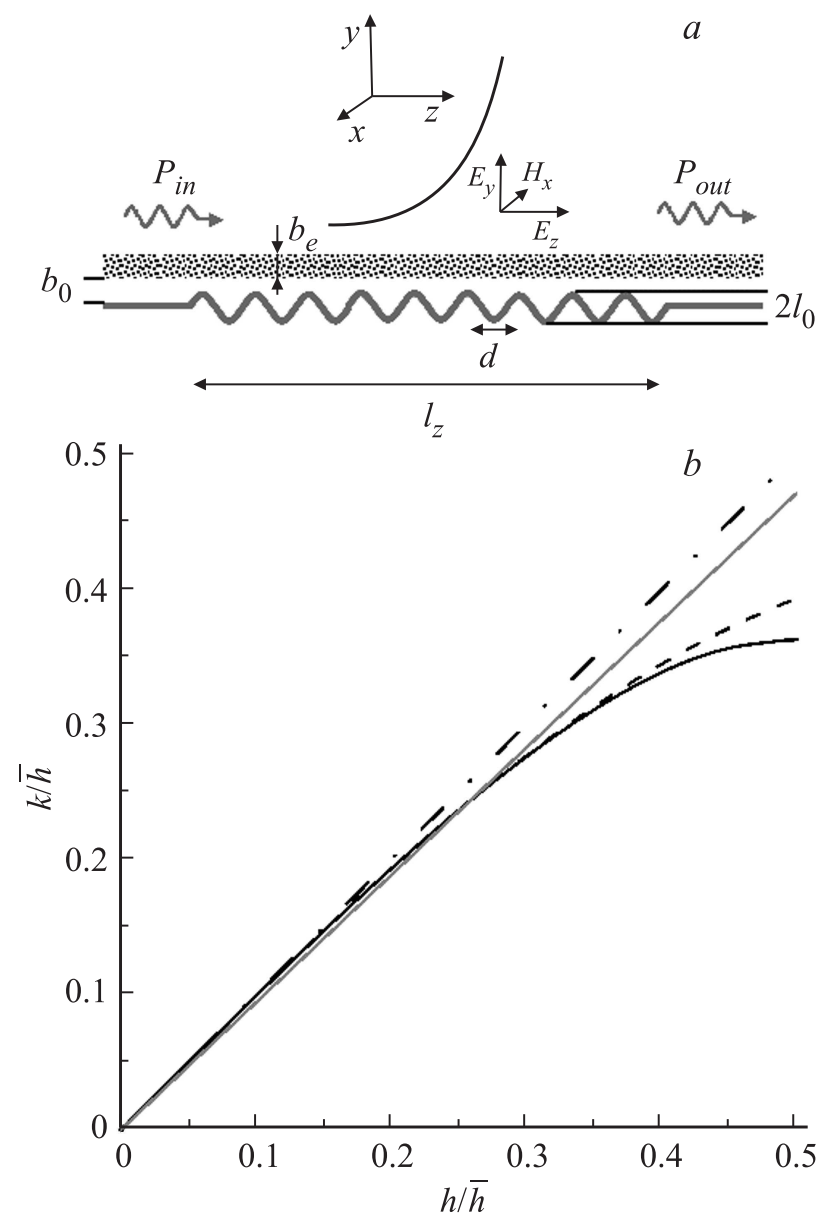

Рис. 1. $a-$ схема усилителя поверхностной волны. $b-$ дисперсионная диаграмма: штрихпунктир - световой конус, сплошная линия - дисперсионная характеристика нормальной поверхностной волны, определяемая уравнением (10) при $\alpha=0.4$, штрихи - то же в импедансном приближении, серая линия - характеристика электронного пучка $(\omega=h v)$.

синусоидально гофрированной металлической поверхностью формы $l(z)=l_{0} \cos (\bar{h} z)$, где $l_{0}$ - амплитуда гофра (рис. 1,a). Поле поверхностной волны, распространяющейся в направлении $z$, обладает ТМ-поляризацией и имеет следующие компоненты:

$$
\begin{gathered}
H_{x}=\operatorname{Re}\left(H_{x}^{\omega}(y, z) e^{i \omega t}\right), \quad E_{y}=\operatorname{Re}\left(E_{y}^{\omega}(y, z) e^{i \omega t}\right), \\
E_{z}=\operatorname{Re}\left(E_{z}^{\omega}(y, z) e^{i \omega t}\right) .
\end{gathered}
$$

Согласно [14], в предположении малой кривизны $\left(l_{0} \ll d\right)$ гофрированная поверхность может быть заменена плоскостью $y=0$, на которой задан эффективный поверхностный магнитный ток

$$
i_{x}^{m \omega}(z)=\left.\frac{c}{4 \pi}\left(\frac{\partial}{\partial z}\left(l(z) E_{y}^{\omega}\right)-i \frac{\omega}{c} l(z) H_{x}^{\omega}\right)\right|_{y=0} .
$$

В рассматриваемой геометрии уравнения Максвелла с учетом поверхностного магнитного тока (2) и объемного продольного электрического тока могут быть представлены в виде

$$
\begin{gathered}
\frac{\partial H_{x}^{\omega}}{\partial z}=i \frac{\omega}{c} E_{y}^{\omega}, \\
\frac{\partial H_{x}^{\omega}}{\partial y}=-i \frac{\omega}{c} E_{z}^{\omega}-\frac{4 \pi}{c} j_{z}^{e \omega}, \\
\frac{\partial E_{y}^{\omega}}{\partial z}-\frac{\partial E_{z}^{\omega}}{\partial y}=i \frac{\omega}{c} H_{x}^{\omega}+\frac{4 \pi}{c} i_{x}^{m \omega} \delta(y)
\end{gathered}
$$

и сведены к одному уравнению Гельмгольца для компоненты $H_{x}$ магнитного поля

$$
\Delta H_{x}^{\omega}+\frac{\omega^{2}}{c^{2}} H_{x}^{\omega}=i \omega \frac{4 \pi}{c^{2}} i_{x}^{m \omega} \delta(y)-\frac{4 \pi}{c} \frac{\partial j_{z}^{e \omega}}{\partial y},
$$

где $\delta(y)-$ дельта-функция, $j_{z}^{e \omega}=\left\langle j_{z}^{e} \exp (-i \omega t)\right\rangle-$ амплитуда гармоники $z$-компоненты объемного электрического тока $j_{z}^{e}$ (угловые скобки означают усреднение по времени).

При $j_{z}^{e}=0$ с учетом периодичности электродинамической системы представим магнитное поле в виде ряда по пространственным гармоникам:

$$
H_{s}^{\omega}=\sum_{s=-\infty}^{+\infty} H_{x}(y, z) e^{i(\omega t-(k+s \bar{h}) z)},
$$

где $\left|\partial H_{x} / \partial z\right| \ll\left|(k+s \bar{h}) H_{x}\right|$. Соответственно компоненты электрического поля определяются выражениями

$$
\begin{gathered}
E_{y}^{\omega}=-\sum_{s=-\infty}^{+\infty} \frac{1}{k}(k+s \bar{h}) H_{s} e^{i(\omega t-(k+s \bar{h}) z)}, \\
E_{z}^{\omega}=\frac{i}{k} \sum_{s=-\infty}^{+\infty} \frac{\partial H_{s}}{\partial y} e^{i(\omega t-(k+s \bar{h}) z)},
\end{gathered}
$$

где $k=\omega / c$. Подставляя разложение полей $(5),(6)$ в уравнение (4) и ограничиваясь в предположении малой глубины гофра учетом только низших $(s=0$ и $s= \pm 1)$ пространственных гармоник, получим, что амплитуды указанных гармоник связаны уравнениями

$$
\begin{gathered}
\frac{\partial H_{0}}{\partial z}+\frac{i}{2 k} \frac{\partial^{2} H_{0}}{\partial y^{2}}=-i \alpha \delta(y)\left[H_{1}-H_{-1}\right], \\
\pm \frac{\partial H_{ \pm 1}}{\partial z}+\frac{i}{2(\bar{h} \pm k)} \frac{\partial^{2} H_{ \pm 1}}{\partial y^{2}}-i \frac{\bar{h}^{2} \pm 2 k \bar{h}}{2(\bar{h} \pm k)} H_{ \pm 1} \\
=\mp i \alpha \frac{k}{\bar{h} \pm k} \delta(y) H_{0},
\end{gathered}
$$

где $\alpha=l_{0} \bar{h} / 4-$ коэффициент связи. Система (7),(8) допускает прижатое к гофрировке решение вида $H_{0, \pm 1}=\hat{H}_{0, \pm 1} e^{-i \Gamma z-g_{0, \pm 1} y}$, соответствующее поверхностной волне. Поперечные декременты пространственных гармоник

$$
g_{0}=\sqrt{2 k \Gamma}, \quad g_{ \pm 1}=\sqrt{(k \pm \bar{h})^{2}-k^{2}+2(k \pm \bar{h}) \Gamma}
$$


связаны дисперсионным уравнением

$$
\Gamma=8 k^{3} \alpha^{4}\left(\frac{1}{g_{1}}+\frac{1}{g_{-1}}\right)^{2} .
$$

Поправка к волновому числу $\Gamma$ характеризует замедление поверхностной волны: $\beta_{p h}=k / h \approx 1-\Gamma / k$. Следующая из уравнения (10) дисперсионная характеристика нормальной волны $\Gamma(k)$ показана на рис. $1, b$ сплошной кривой.

В работах [6,7] были исследованы генерационные режимы, которые реализуются при частотах излучения, близких к брэгговской частоте $k \approx \bar{h} / 2$, когда поле излучения представляется как комбинация попутного $H_{0}$ и встречного $H_{-1}$ квазиоптических пучков. В настоящей работе рассматривается режим усиления, для реализации которого частота падающей волны должна быть далека от брэгговского резонанса во избежание самовозбуждения (рис. 1, $b$ ). В этом случае декременты поперечного спадания (9) можно приближенно переписать в виде

$$
g_{ \pm 1} \approx \sqrt{(k+\bar{h})^{2}-k^{2}}
$$

Соответственно в уравнениях (8) можно пренебречь производными по продольной координате, записав решение этих уравнений в явном виде

$$
H_{ \pm 1}=\hat{H}_{ \pm 1} e^{-g_{ \pm 1} y}, \quad \text { где } \quad \hat{H}_{ \pm 1}= \pm 2 \alpha \frac{k}{g_{ \pm 1}} H_{0} .
$$

В результате система уравнений (7), (8) сводится к одному уравнению

$$
\frac{\partial H_{0}}{\partial z}+\frac{i}{2 k} \frac{\partial^{2} H_{0}}{\partial y^{2}}+i \chi \delta(y) H_{0}=0 .
$$

Здесь

$$
\chi=2 \alpha^{2} \Phi(k), \text { где } \Phi(k)=\frac{k}{\sqrt{(k+\bar{h})^{2}-k^{2}}}+\frac{k}{\sqrt{(k-\bar{h})^{2}-k^{2}}} .
$$

Данное приближение будем далее называть импедансным [10], а величину $\chi$ - поверхностным импедансом, поскольку из уравнения (13) следует, что связь между электрическим и магнитным полем на поверхности имеет локальный характер:

$$
\left.\frac{i}{2 k} \frac{\partial H_{0}}{\partial y}\right|_{y=0}=\left.i \chi H_{0}\right|_{y=0} \text {, т. е. } E_{z}^{\omega}=-2 i \chi H_{x}^{\omega} .
$$

В описанном приближении дисперсионное уравнение (10) упрощается и принимает вид функциональной зависимости

$$
\Gamma(k)=2 k \chi(k)^{2} .
$$

При этом поперечный декремент спадания нулевой гармоники пропорционален величине импеданса: $g_{0}=2 k \chi$. На рис. $1, b$ при $\alpha=0.4$ сплошной кривой показана

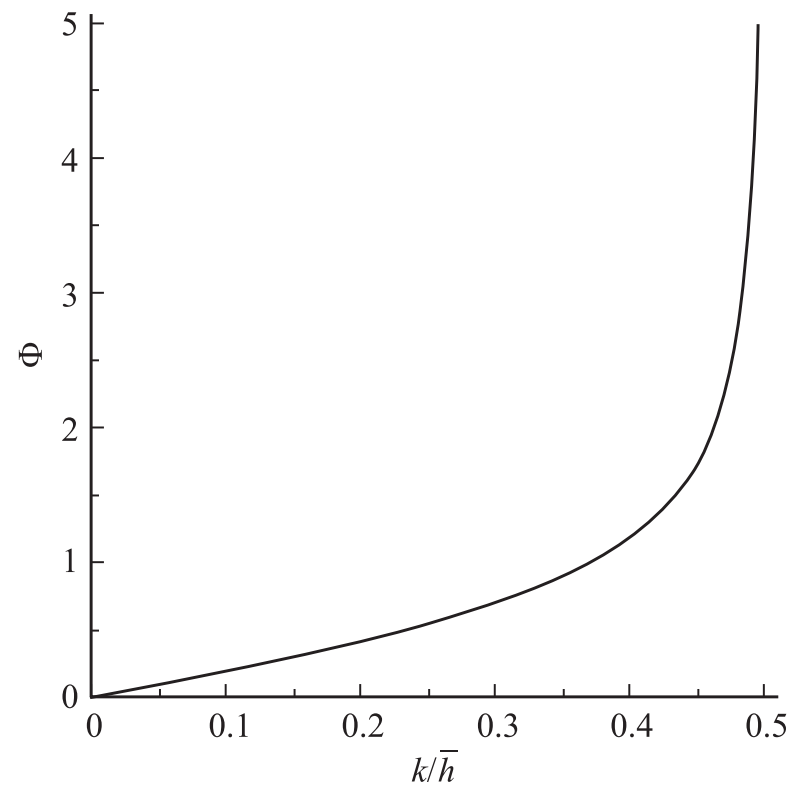

Рис. 2. Импеданс как функция частоты.

дисперсионная характеристика нормальной поверхностной волны, определяемая уравнением (10), а пунктиром показана такая же характеристика, полученная в рамках импедансного приближения (уравнение (16)). При этом функция $\Phi(k)$, определяющая зависимость величины импеданса от частоты, приведена на рис. 2. Очевидно, что импедансное приближение справедливо вдали от брэгговского резонанса и нарушается, когда частота излучения становится близка к брэгговской частоте $k \approx \bar{h} / 2$, а импеданс $\chi \sim \Phi(k) \rightarrow \infty$.

\section{2. Самосогласованные уравнения, описывающие усиление поверхностной волны релятивистским электронным потоком}

Рассмотрим синхронное черенковское взаимодействие релятивистского потока электронов с поверхностной волной. Считаем, что электроны движутся параллельно гофрированной поверхности вдоль направления ведущего магнитного поля с продольной скоростью $v=\beta c$. В этих условиях группировка частиц происходит под действием продольной компоненты электрического поля, которая, согласно (3), равна

$$
E_{z}=\operatorname{Re}\left(\frac{i}{k}\left(\frac{\partial H_{0}}{\partial y}+\exp [i k z] \frac{4 \pi}{c} j_{z}^{e \omega}\right) e^{i(\omega t-k z)}\right) .
$$

Важно подчеркнуть, что в случае ленточных электронных пучков малой толщины и большой плотности продольная компонента наводимого в электронном пучке высокочастотного тока оказывает существенное влияние на величину действующего поля. 
Продольное движение и группировка релятивистских частиц описывается уравнениями [7]

$$
\begin{gathered}
\frac{\partial \gamma}{\partial z}=-\frac{e}{m c^{2}} E_{z}, \\
\frac{\partial \theta}{\partial z}=k\left(\frac{1}{\beta(\gamma)}-1\right),
\end{gathered}
$$

где $m c^{2} \gamma\left(y, z, \theta_{0}\right)$ - энергия электрона, $\theta\left(y, z, \theta_{0}\right)-$ его фаза в поле синхронной волны, $\beta(\gamma)=\left(1-\gamma^{-2}\right)^{1 / 2}$.

В приближении относительно малого изменения энергии частиц $\left(\gamma-\gamma_{0}\right) / \gamma_{0} \ll 1$ уравнения движения (18) упрощаются и трансформируются к виду

$$
\frac{\partial^{2} \theta}{\partial z^{2}}=\frac{\mu e}{m c^{2} \gamma_{0}} \operatorname{Re}\left(i\left(\frac{\partial H_{0}}{\partial y}+\exp [i k z] \frac{4 \pi}{c} j_{z}^{e \omega}\right) \exp i \theta\right),
$$

где $\mu=\gamma_{0}^{-2} \beta_{0}^{-3}-$ параметр инерционной группировки. В случае первоначально стационарного электронного пучка, равномерно распределенного по фазам влета, граничные условия к уравнению (19) имеют вид

$$
\left.\theta\right|_{z=0}=\theta_{0} \in[0,2 \pi),\left.\frac{\partial \theta}{\partial z}\right|_{z=0}=\Delta \text {, где } \Delta=k \frac{\left(1-\beta_{0}\right)}{\beta_{0}} \text {. }
$$

Для описания возбуждения электронным пучком электродинамической системы в уравнении (13) следует учесть синхронную гармонику $j_{z}^{e \omega}$ объемного электронного тока в соответствии с (4). Электронный ток, наводимый в пучке в результате взаимодействия с электромагнитным полем, представляет собой периодическую функцию времени с периодом $T=2 \pi / \omega$. Соответственно ток может быть разложен в ряд Фурье

$$
j_{z}^{e}=j_{0}+\operatorname{Re} \sum_{n=-\infty}^{+\infty} j_{z}^{e n \omega} e^{i n \omega t}
$$

где для гармоник с учетом закона сохранения заряда имеем

$$
j_{z}^{e n \omega}=\frac{1}{\pi} \int_{0}^{2 \pi} j_{z}^{e} e^{-i n \omega t} d \omega t=\frac{j_{0}}{\pi} \int_{0}^{2 \pi} e^{-i n \omega t} d \omega t_{0} .
$$

Невозмущенное распределение тока по поперечной координате $y$ представим в виде $j_{0}=-I_{0} f(y) / b_{e}$, где $I_{0}-$ погонный ток пучка, $f(y)$ - функция, описывающая распределение тока, $b_{e}=\int_{0}^{\infty} f(y) d y-$ эффективная толщина пучка.

Учитывая, согласно (22), объемный электрический ток $j_{z}^{e \omega} \neq 0$ в уравнении (4) и представляя его решение в виде (5), приведем уравнение для основной гармоники поля в импедансном приближении к виду

$$
\frac{\partial H_{0}}{\partial z}+\frac{i}{2 k} \frac{\partial^{2} H_{0}}{\partial y^{2}}+i \chi \delta(y) H_{0}=i \frac{2 \pi I_{0}}{k c} \frac{1}{b_{e}} \frac{\partial}{\partial y}(f(y) J)
$$

(ср. с (13)), где $J=\pi^{-1} \int_{0}^{2 \pi} \exp [-i \theta] d \theta_{0}-$ амплитуда высокочастотного электронного тока, определяемая на основе решения уравнений движения (19).
Для уменьшения числа независимых параметров в уравнениях (19) и (23) целесообразно ввести параметр усиления

$$
G=\left(2 \sqrt{2} \frac{e I_{0}}{m c^{3}} \frac{\mu}{\gamma_{0}} \lambda\right)^{2 / 3}
$$

являющийся аналогом параметра Пирса [7,9], и нормировать на него остальные переменные и параметры:

$$
\begin{gathered}
Z=G k z, \quad Y=\sqrt{2 G} k y, \quad B_{e}=\sqrt{2 G} k b_{e}, \quad \hat{\chi}=\sqrt{\frac{2}{G}} \chi, \\
C=\frac{\sqrt{2} e \mu}{m c^{2} \gamma_{0} k G^{3 / 2}} H_{0}, \quad F(Y)=f(y) .
\end{gathered}
$$

В введенных переменных самосогласованная система уравнений (19), (23) с граничными условиями (20) перепишется в виде

$$
\begin{gathered}
\frac{\partial C}{\partial Z}+i \frac{\partial^{2} C}{\partial Y^{2}}+i \hat{\chi} \delta(Y) C=i \frac{1}{B_{e}} \frac{\partial}{\partial Y}(F(Y) J), \\
\frac{\partial^{2} \theta}{\partial Z^{2}}=\operatorname{Re}\left(i \frac{\partial C}{\partial Y} e^{i \theta}-i \frac{1}{B_{e}}(F(Y) J) e^{i \theta}\right), \\
\left.\theta\right|_{Z=0}=\theta_{0} \in[0,2 \pi),\left.\quad \frac{\partial \theta}{\partial Z}\right|_{Z=0}=\hat{\Delta}, \text { где } \hat{\Delta}=\Delta / k G .
\end{gathered}
$$

Для описания усиления первоначально стационарным немодулированным электронным потоком внешнего сигнала, который представляет собой квазиоптический волновой пучок, падающий на систему в сечении $Z=0$ (рис. 1), граничные условия (28) к уравнениям (26), (27) должны быть дополнены условием для поля

$$
C_{Z=0}=C_{0}(Y) \text {. }
$$

Электронный КПД (относительная доля кинетической мощности электронного потока, преобразующаяся в излучение) определяется соотношениями

$$
\eta=\frac{G \hat{\eta}}{\mu\left(1-\gamma_{0}^{-1}\right)}
$$

где

$$
\hat{\eta}=\left.\frac{1}{2 \pi B_{e}} \int_{0}^{B} \int_{0}^{2 \pi}\left(\frac{\partial \theta}{\partial Z}-\hat{\Delta}\right)\right|_{Z=L} F(Y) d \theta_{0} d Y
$$

- приведенный КПД, $L=G k l_{z}$.

Из уравнений (26), (27) следует закон сохранения энергии в системе „Пучок-волна“

$$
\hat{P}(Z)=\hat{P}(0)=4 \hat{\eta},
$$

где $\hat{P}=\int_{0}^{\infty}|C|^{2} d Y-$ нормированная мощность, переносимая волной. В реальных переменных мощность излучения выражается соотношением

$$
P=\frac{1}{8 \sqrt{2 \lambda}}\left(\frac{m^{2} c^{5}}{e^{2}}\right)\left(\frac{\gamma_{0}}{\mu}\right)^{2} G^{5 / 2} \hat{P} .
$$

Коэффициент усиления $K$ определяется следующим образом: $K=\hat{P}(Z) / \hat{P}(0)$. 


\section{3. Приближение малого сигнала. Дисперсионное уравнение}

В приближении малого сигнала линеаризуем уравнения движения частиц (27). Считая смещения фазы электронов малыми $\theta=\theta_{0}+\vartheta,|\vartheta| \ll 1$, электрический объемный ток в правой части уравнения возбуждения можно переписать в виде $J=-i \pi^{-1} \int_{0}^{2 \pi} \vartheta \exp \left[-i \theta_{0}\right] d \theta_{0}$. Тогда, интегрируя (27) по фазам влета $\theta_{0}$, получим систему уравнений, описывающую линейную стадию усиления:

$$
\begin{gathered}
\frac{\partial C}{\partial Z}+i \frac{\partial^{2} C}{\partial Y^{2}}+i \hat{\chi} \delta(Y) C=i \frac{1}{B_{e}} \frac{\partial}{\partial Y}(F(Y) J) \\
\left(\frac{\partial}{\partial Z}+i \hat{\Delta}\right)^{2} J=\frac{\partial C}{\partial Y}-\frac{1}{B_{e}} F(Y) J
\end{gathered}
$$

Заметим, что последний член в правой части уравнения (34) обусловлен влиянием пространственного заряда электронного пучка. Этот член возникает при учете продольной компоненты электрического поля в уравнении для действующего поля (17).

На основании линейной системы уравнений (33), (34) получим дисперсионное уравнение для системы: ленточный электронный пучок, невозмущенная плотность которого однородна по всей толщине $B_{e}$, движется над безграничной по продольной координате $Z$ импедансной поверхностью,

$$
F(Y)=\left\{\begin{array}{ll}
1, & Y \in\left[B_{0}, B_{0}+B_{e}\right] \\
0, & Y \notin\left[B_{0}, B_{0}+B_{e}\right]
\end{array} .\right.
$$

Представив функции в виде $C, J=\tilde{C}, \tilde{J} \exp [-i \hat{\Gamma} Z]$, из уравнения (34) имеем

$$
\tilde{J}=-\frac{1}{(\hat{\Delta}-\hat{\Gamma})^{2}-B_{e}^{-1} F(Y)} \frac{d \tilde{C}}{d Y}
$$

С учетом (36) система уравнений (33), (34) преобразуется к одному уравнению

$$
\begin{aligned}
-\hat{\Gamma} \tilde{C} & +\frac{d^{2} \tilde{C}}{d Y^{2}}+\hat{\chi} \delta(Y) \tilde{C}=-\frac{1}{B_{e}} \frac{d}{d Y} \\
& \times\left(\frac{1}{\left[(\hat{\Delta}-\hat{\Gamma})^{2}-B_{e}^{-1} F(Y)\right]} F(Y) \frac{d \tilde{C}}{d Y}\right),
\end{aligned}
$$

из которого следуют граничные условия для магнитного поля на краях пучка и на гофрированной поверхности. Принимая во внимание форму распределения тока (35), получим упрощенный вид граничных условий (здесь $\varepsilon \rightarrow 0)$

$$
\left.\left(\frac{d \tilde{C}}{d Y}+\tilde{\chi} \tilde{C}\right)\right|_{Y=0}=0
$$

$$
\left.\frac{d \tilde{C}}{d Y}\right|_{Y=B_{0}+\varepsilon}-\left.\frac{d \tilde{C}}{d Y}\right|_{Y=B_{0}-\varepsilon}=-\left.\frac{1}{B_{e}} \frac{1}{(\hat{\Delta}-\hat{\Gamma})^{2}-B_{e}^{-1}} \frac{d \tilde{C}}{d Y}\right|_{Y=B_{0}+\varepsilon}
$$

$$
\begin{aligned}
\left.\frac{d \tilde{C}}{d Y}\right|_{Y=B_{0}+B_{e}+\varepsilon} & -\left.\frac{d \tilde{C}}{d Y}\right|_{Y=B_{0}+B_{e}-\varepsilon} \\
= & \left.\frac{1}{B_{e}} \frac{1}{(\hat{\Delta}-\hat{\Gamma})^{2}-B_{e}^{-1}} \frac{d \tilde{C}}{d Y}\right|_{Y=B_{0}+B_{e}-\varepsilon} .
\end{aligned}
$$

Первое условие (38) представляет собой импедансное граничное условие на поверхности идеального металла и фактически учитывает гофрировку поверхности. Условия (39), (40) описывают разрывы производной магнитного поля на границах электронного пучка. При этом действующее на электроны продольное электрическое поле $E_{z} \propto \partial \tilde{C} / \partial Y-B_{e}^{-1}(F(Y) \tilde{J})$ на границе пучка конечной плотности остается непрерывным.

Представляя решения уравнения (37) вовне и внутри электронного пучка в виде

$$
\tilde{C}(Y)= \begin{cases}C_{1} e^{-\hat{g} Y}, & Y>B_{0}+B_{e}, \\ C_{2} e^{-\kappa Y}+C_{3} e^{\kappa Y}, & Y \in\left[B_{0}, B_{0}+B_{e}\right], \\ C_{4} e^{-\hat{g} Y}+C_{5} e^{\hat{g} Y}, & Y \in\left(0, B_{0}\right),\end{cases}
$$

где

$$
\hat{g}=\sqrt{\hat{\Gamma}}, \quad \kappa=\sqrt{\frac{\hat{\Gamma}\left(B_{e}(\hat{\Delta}-\hat{\Gamma})^{2}-1\right)}{B_{e}(\hat{\Delta}-\hat{\Gamma})^{2}}}
$$

- соответствующие комплексные поперечные декременты. С учетом граничных условий (38)-(40) получим дисперсионное уравнение в общей форме

$$
\begin{aligned}
\kappa[v & -1]\left(1+\frac{1}{B_{e}(\hat{\Delta}-\hat{\Gamma})^{2}-1}\right) \\
& +\hat{g} \frac{\left((\hat{g}+\hat{\chi}) e^{-\hat{g} B_{0}}-(\hat{g}-\hat{\chi}) e^{\hat{g} B_{0}}\right)}{\left((\hat{g}+\hat{\chi}) e^{-\hat{g} B_{0}}+(\hat{g}-\hat{\chi}) e^{\hat{g} B_{0}}\right)}[v+1]=0,
\end{aligned}
$$

где

$$
v=\frac{(\kappa-\hat{g})\left(B_{e}(\hat{\Delta}-\hat{\Gamma})^{2}-1\right)+\kappa}{(\kappa+\hat{g})\left(B_{e}(\hat{\Delta}-\hat{\Gamma})^{2}-1\right)+\kappa} e^{-2 \kappa B_{e}} .
$$

В случае предельно тонкого электронного слоя $\kappa B_{e} \ll 1$ запишем $v \approx-1-2 \kappa B_{e}\left((\hat{\Delta}-\hat{\Gamma}) \hat{g}^{-1}-1\right)$, тогда дисперсионное уравнение (43) редуцируется к виду

$$
(\hat{\Delta}-\hat{\Gamma})^{2}(\hat{g}-\hat{\chi})=-\frac{\hat{g}}{2} e^{-\hat{g} B_{0}}\left((\hat{g}+\hat{\chi}) e^{-\hat{g} B_{0}}-(\hat{g}-\hat{\chi}) e^{\hat{g} B_{0}}\right) .
$$

При анализе дисперсионных уравнений (43) и (45) следует заметить, что продольные и поперечные волновые числа при учете взаимодействия с электронным пучком являются, вообще говоря, комплексными. В общем 
случае эти уравнения допускают счетное множество решений. С точки зрения усиления представляют интерес решения, удовлетворяющие следующим критериям:

$$
\operatorname{Re} \hat{g}>0 \quad \text { и } \quad \operatorname{Im} \hat{g}>0 .
$$

Такие решения соответствуют прижатой моде, амплитуда которой спадает по оси $y$ при удалении от пучка и гофрированной поверхности и которая имеет поток энергии, направленный от пучка к периферии. Поскольку $\hat{\Gamma}=\hat{g}^{2}$, такая мода обладает положительным продольным инкрементом

$$
\operatorname{Im}(\hat{\Gamma})=2 \operatorname{Im}(\hat{g}) \operatorname{Re}(\hat{g})>0 .
$$

В случае тонкого ленточного электронного пучка усиление описывается уравнением (45), которое содержит три независимых нормированных параметра: $\hat{\Delta}, \hat{\chi}$ и $B_{0}$. На рис. 3 представлены наименьшие по абсолютному значению корни уравнения (45). Отметим, что лишь

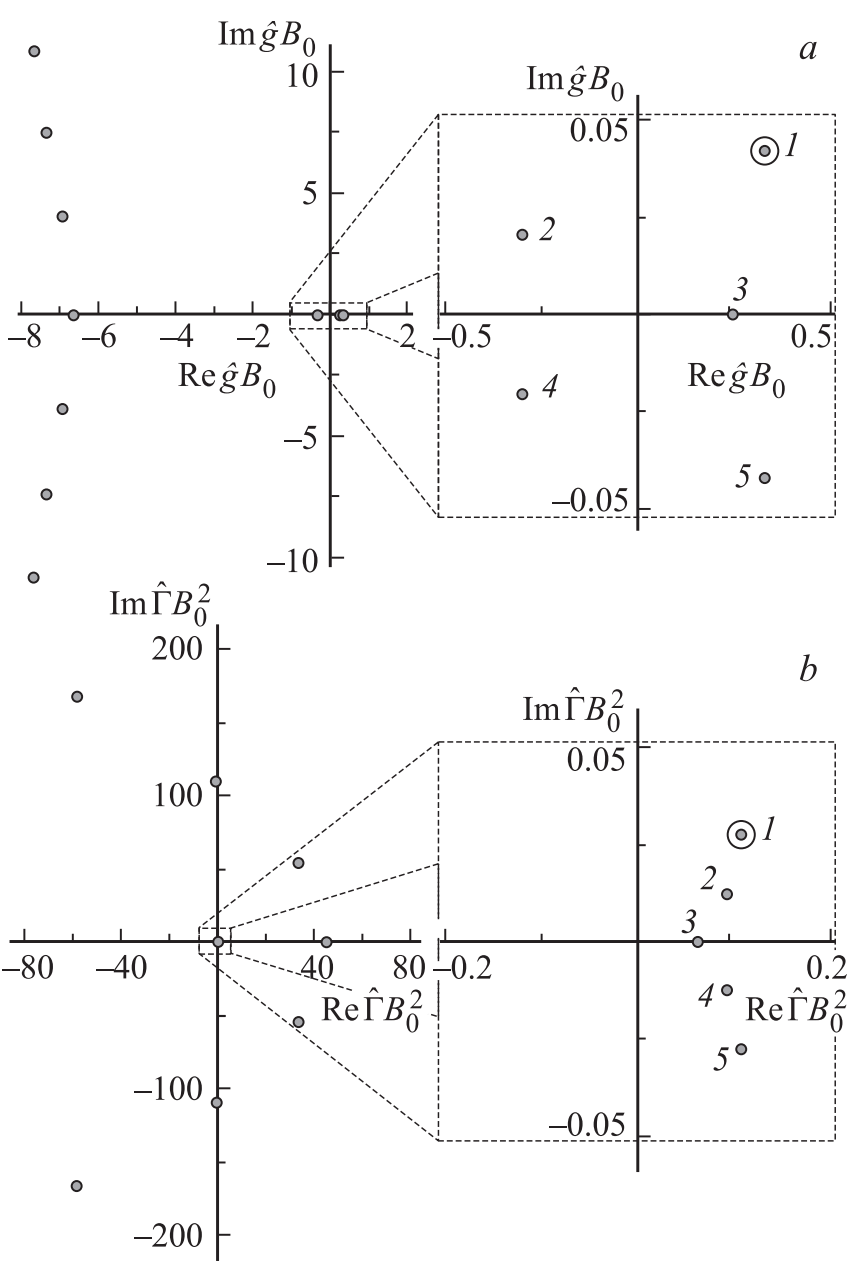

Pис. 3. Наименьшие по абсолютному значению корни уравнения (45). $a-$ нормированные поперечные волновые числа решений, $b$ - поправка к продольному волновому числу. Единственное решение, удовлетворяющее условиям (46), (47), соответствует номеру $1 ; \hat{\chi}=3, \hat{\Delta}=9, B_{0}=0.1$.
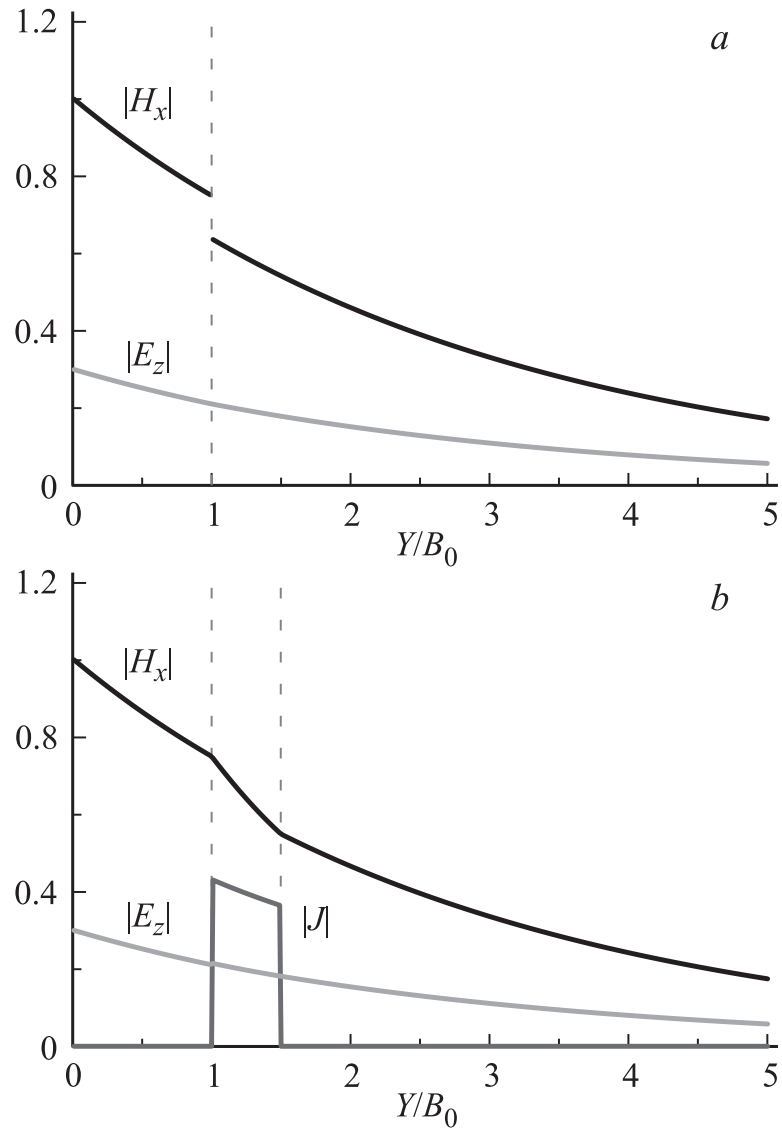

Рис. 4. Поперечные структуры модулей амплитуд магнитного поля $H_{x}$ и действующей на электроны продольной компоненты электрического поля $E_{z}$ для случая $(a)$ ленточного электронного пучка, движущегося на расстоянии $B_{0}=0.1$ от импедансной поверхности, $(b)$ электронного пучка конечной ширины $B_{e}=0.05$, движущегося на том же расстоянии от поверхности, $\hat{\chi}=3, \hat{\Delta}=9$.

один из них, обозначенный индексом „1““ на врезках с увеличенным масштабом, удовлетворяет условиям (46), (47). Все прочие корни, которые по абсолютной величине превосходят отображенные на рис. 3 , расположены в левой комплексной полуплоскости $\operatorname{Re} \hat{g}<0$. Таким образом, в данном случае существует единственное решение, нарастающее по продольной координате $Z$ и спадающее по поперечной (нормальной) координате $Y$. Распределения амплитуд магнитного поля и действующей на электроны продольной компоненты электрического поля для этого решения приведены на рис. 4, $a$. Очевидно, что в случае ленточного электронного пучка и наводимого в нем продольного поверхностного тока магнитное поле $H_{x}$ в соответствии с уравнениями Максвелла испытывает скачок, в то время как действующее на частицы продольное электрическое поле $E_{z}$ остается непрерывным.

Следует отметить, что в случае больших нормированных значений импеданса $\hat{\chi}$, которым фактически соответствуют малые значения тока инжекции, можно 

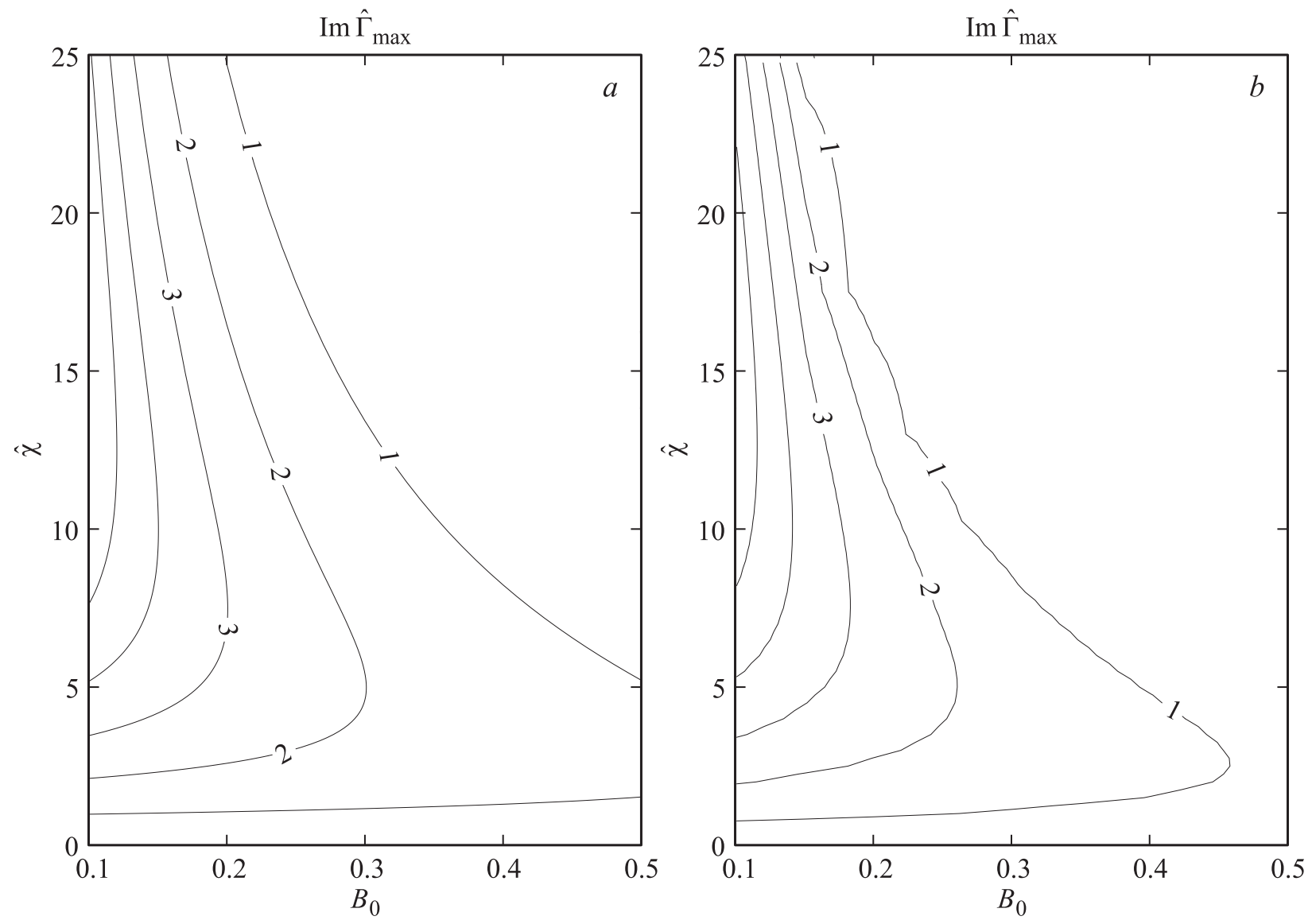

Рис. 5. $a$ - линии уровня продольного инкремента $\operatorname{Im}(\hat{\Gamma})$, полученные в приближении фиксированной поперечной структуры поля в условиях точного синхронизма $\hat{\Delta}=\hat{\chi}^{2} ; b-$ то же в рамках модели с нефиксированной структурой поля (45).

представить решение дисперсионного уравнения (45) в виде

$$
\hat{g}=\hat{\chi}-\xi
$$

где $|\xi| \ll \hat{\chi}, B_{0}^{-1}$. С учетом (48) уравнение (45) преобразуется к канонической для черенковских усилителей типа „О“ форме (см. [7,9])

$$
\left(\Delta_{\text {eff }}-\hat{\xi}\right)^{2} \hat{\xi}=1,
$$

где $\quad \Delta_{\text {eff }}=2^{-1 / 3} \hat{\chi}^{-1} \exp \left[4 \hat{\chi} B_{0} / 3\right]\left(\hat{\chi}^{2}-\hat{\Delta}\right), \quad \hat{\xi}=2^{2 / 3} \times$ $\times \exp \left[2 \hat{\chi} B_{0} / 3\right] \xi$. Заметим, что представление (48) соответствует приближению фиксированной поперечной структуры поля. В рамках данного приближения поперечный (по оси $Y$ ) профиль поля излучения считается совпадающим со структурой прижатой моды (13), распространяющейся вдоль гофрированной импедансной поверхности в отсутствие электронного пучка.

Как известно[7,9], в рамках уравнений (49) максимум инкремента $\operatorname{Im}(\hat{\xi})_{\max }=\sqrt{3} / 2$ достигается при $\Delta_{\text {eff }}=0$, т. е. при $\hat{\Delta}=\hat{\chi}^{2}$. В физических переменных это условие соответствует совпадению невозмущенной поступательной скорости частиц с фазовой скоростью замедленной волны. Соответственно инкремент определяется соотно- шением

$$
\begin{aligned}
\operatorname{Im}(\Gamma)_{\max } & =G k \operatorname{Im}(\hat{\Gamma})_{\max } \\
& =\frac{\sqrt{3}}{2} \frac{2 G k}{2^{2 / 3} e^{2 \hat{\chi} B_{0} / 3}}\left(\sqrt{\frac{2}{G}} \chi+\frac{1}{2^{5 / 3} e^{2 \hat{\chi} B_{0} / 3}}\right) \\
& \approx \sqrt{3} k \chi e^{\frac{-2 \hat{\chi} B_{0}}{3}}\left(2 \frac{e I_{0}}{m c^{3}} \frac{\mu}{\gamma_{0}} \lambda\right)^{1 / 3}
\end{aligned}
$$

Очевидно, что максимального значения

$$
\operatorname{Im}(\Gamma)_{\max }=\frac{3 \sqrt{3}}{4 b_{0}} e^{-1}\left(2 \frac{e I_{0}}{m c^{3}} \frac{\mu}{\gamma_{0}} \lambda\right)^{\frac{1}{3}}
$$

инкремент будет достигать при выполнении условия $\hat{\chi}=1.5 B_{0}^{-1}$, которое фактически определяет оптимальную величину поверхностного импеданса при фиксированной величине зазора между пучком и замедляющей структурой.

На рис. 5, $a$ изображены линии уровня нормированного значения инкремента $\operatorname{Im}(\hat{\Gamma})$ на плоскости параметров $\left(\hat{\chi}, B_{0}\right)$, полученного в приближении фиксированной структуры поля на основании формулы (50). Для сравнения на рис. 5, $b$ представлены те же линии, 

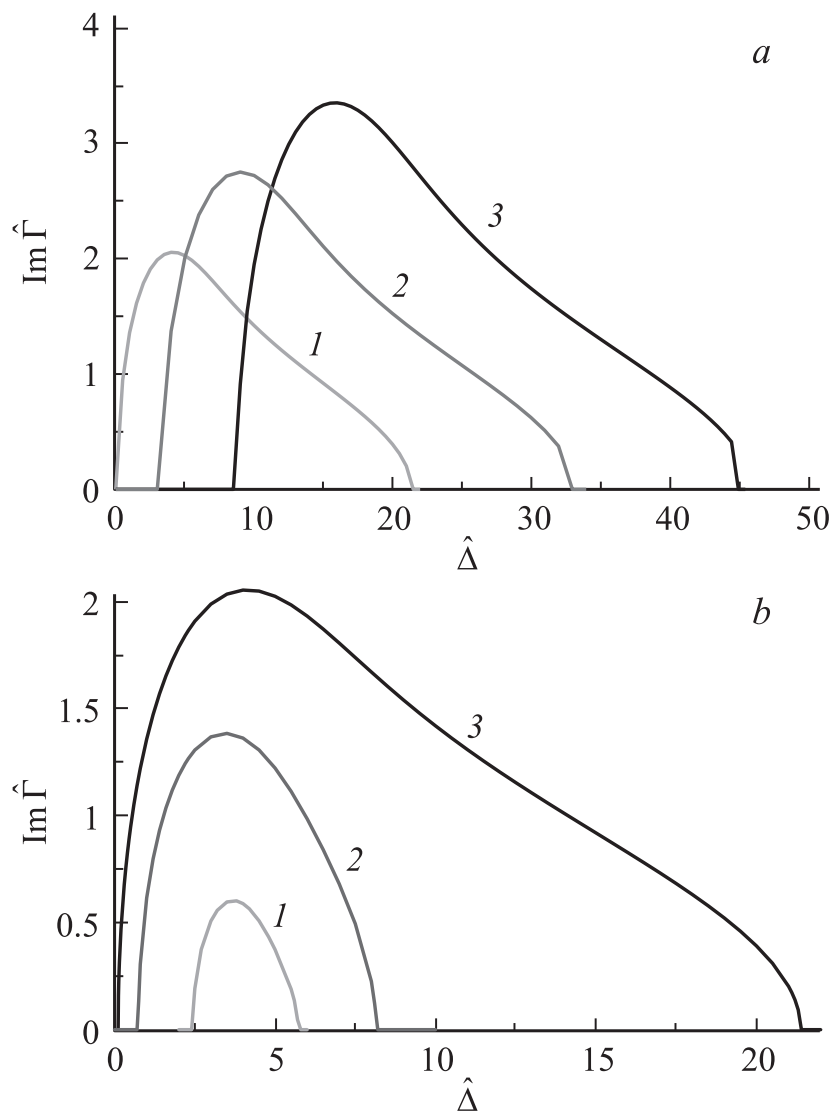

Рис. 6. $a-$ зависимости продольного инкремента $\operatorname{Im}(\hat{\Gamma})$ от величины $\hat{\Delta}$ для различных значений импеданса: $1-\hat{\chi}=2$, $2-\hat{\chi}=3,3-\hat{\chi}=4$ (зазор между ленточным пучком и импедансной поверхностью фиксирован $\left.B_{0}=0.1\right) . b-$ то же, но при фиксированном импедансе $\hat{\chi}=2$ и различных величинах зазора: $1-B_{0}=0.5,2-B_{0}=0.3,3-B_{0}=0.1$.

полученные путем численного решения уравнения (45) в условиях точного электронноволнового синхронизма $\hat{\Delta}=\hat{\chi}^{2}$. Найденные зависимости имеют аналогичный характер и сравнительно небольшие (менее $10 \%$ по абсолютному значению) расхождения в области оптимальных по инкременту нормированных параметров. Таким образом, для приближенных практических оценок инкремента при больших значениях нормированного импеданса можно пользоваться формулой (50).

На рис. 6, $a$ для трех различных значений импеданса $\hat{\chi}$ приведены зависимости продольного инкремента от величины $\hat{\Delta}$ при фиксированном зазоре $B_{0}=0.1$. В целом зависимость инкремента от величины $\hat{\Delta}$, т.е. от отличия поступательной скорости частиц от скорости света, аналогична имеющей место в приближении фиксированной структуры поля. В частности, при больших значениях импеданса максимум инкремента достигается в условиях точного синхронизма $\hat{\Delta}=\hat{\chi}^{2}$. Имеется срыв режима усиления (исчезает решение, удовлетворяющее условиям (46)) в области малых значений $\hat{\Delta}$, т. е. когда электроны сильно обгоняют волну. С другой стороны, срыв возникает и в области больших $\hat{\Delta}$, когда поступательная скорость электронов становится значительно меньше фазовой скорости замедленной волны. Здесь важно подчеркнуть, что в приближении фиксированной структуры поля срыв усиления в области больших $\hat{\Delta}$ отсутствует.

На рис. 6, $b$ приведены зависимости продольного инкремента от $\hat{\Delta}$ при фиксированном нормированном импедансе $\hat{\chi}=2$ для трех различных значений зазора $B_{0}$. Видно, что по мере удаления электронного потока от поверхности продольный инкремент падает, и область положительных инкрементов сужается, стремясь к окрестности точного резонанса $\hat{\Delta}=4$.

Таким образом, учет нефиксированности поперечной структуры поля позволяет описать порог возникновения усиления в области больших значений $\hat{\Delta}$ (т. е. в области относительно малых скоростей), который отсутствует в приближении фиксированной структуры поля. Заметим, что для традиционных моделей ЛБВ типа „О“ подобный порог возникает при учете влияния полей собственного пространственного заряда электронного пучка. В случае предельно малого зазора $B_{0} \rightarrow 0$ пороговое значение $\hat{\Delta}$ стремится к бесконечности, что может быть проинтерпретировано как следствие взаимной компенсации полей собственного заряда пучка его отражением на металлической поверхности идеального проводника.

На рис. 7 представлены зависимости продольных инкрементов от нормированной расстройки $\hat{\Delta}$ в условиях точного синхронизма $\hat{\Delta}=\hat{\chi}^{2}$, полученные в результате численного решения уравнений (43) и (44) при фиксированном зазоре между нижней границей электронного пучка и импедансной поверхностью $B_{0}=0.1$, но при различных толщинах пучка: $B_{e}=0.1,0.05$ и 0.01 . Как

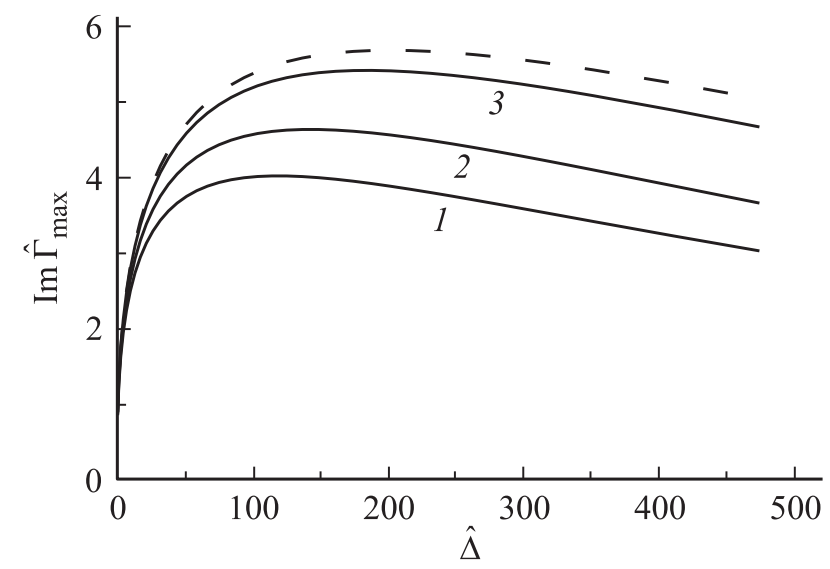

Рис. 7. Зависимости продольного инкремента от параметpa $\hat{\Delta}$ в условиях точного синхронизма $\left(\hat{\Delta}=\hat{\chi}^{2}\right)$. Зазор между нижней границей электронного пучка и импедансной поверхностью фиксирован $B_{0}=0.1$, но меняется толщина пучка: $1-B_{e}=0.1,2-B_{e}=0.05,3-B_{e}=0.001$. Штриховая кривая - та же зависимость для модели бесконечно тонкого ленточного пучка. 
и следовало ожидать, при уменьшении толщины пучка инкремент растет и при выполнении условия $\kappa B_{e} \ll 1$ достигает описываемого уравнением (45) предела, соответствующего случаю бесконечно тонкого ленточного пучка. Поперечные структуры модулей амплитуд магнитного поля, высокочастотного тока и действующей на электроны компоненты электрического поля изображены на рис. $4, b$.

\section{4. Нелинейный режим усиления}

Для моделирования нелинейной стадии процесса параболическое уравнение (26) следует дополнить граничными условиями для поля в области $Y>0$. В работе [12] в качестве таких условий использовалось обращение в ноль продольной компоненты электрического поля на регулярной пластине, установленной на определенном расстоянии от гофрированной поверхности. В настоящей работе в предположении, что какие-либо электродинамические структуры в области $Y>0$ отсутствуют,
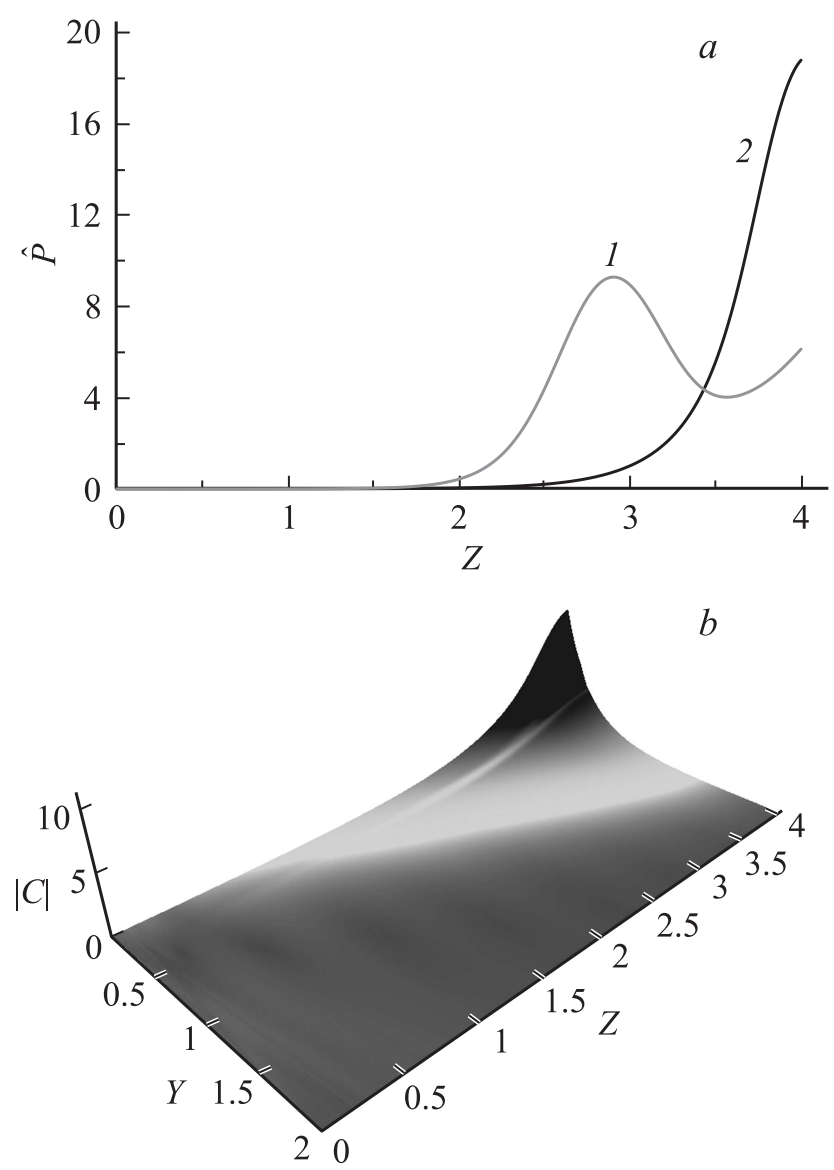

Рис. 8. $a-$ зависимость нормированной мощности усиливаемой волны от продольной координаты в режиме, соответствующем максимуму инкремента $\hat{\Delta}=12.25$ (кривая 1), и в режиме, соответствующем максимуму КПД $\hat{\Delta}=7$ (кривая 2 ). $b$ - пространственное распределение амплитуды усиливаемой волны при $\hat{\Delta}=7 ; \hat{\chi}=3.5, B_{0}=0.1$. использовалось условие излучения [15]

$$
\left.\left(C+\frac{1}{\sqrt{\pi i}} \int_{0}^{Z} \frac{1}{\sqrt{Z-Z^{\prime}}} \frac{\partial C}{\partial Y} d Z^{\prime}\right)\right|_{Y=B}=0,
$$

которое ставилось в некоторой плоскости $Y=B>B_{0}$ $+B_{e}$. При этом предполагалось, что падающее при $Z=0$ (усиливаемое) поле представляет собой волновой пучок, заключенный внутри области $0<Y<B$.

На рис. 8 представлены результаты моделирования уравнений (26)-(29) при различных значениях эффективной расстройки синхронизма. В соответствии с анализом линейного режима максимум инкремента достигается в условиях точного синхронизма $\hat{\Delta}=\hat{\chi}^{2}$. Вместе с тем максимум КПД и мощности усиленного излучения реализуется в области $\hat{\Delta}<\hat{\chi}^{2}$, т. е. в условиях, когда поступательная скорость электронов превосходит фазовую скорость замедленной волны на заданной частоте. В целом указанные зависимости являются типичными для черенковских ЛБВ-усилителей [7,9]. Характерное пространственное распределение поля усиливаемой волны показано на рис. $8, b$. В процессе усиления волна остается прижатой к поверхности замедляющей системы.

Проанализируем на основании развитой теории возможность реализации релятивистского усилителя на длину волны $0.9 \mathrm{~mm}$. Пусть энергия электронов $1 \mathrm{MeV}$, погонная плотность тока $500 \mathrm{~A} / \mathrm{cm}$, период гофрировки $0.25 \mathrm{~mm}$, ее амплитуда $0.06 \mathrm{~mm}$, расстояние от гофрировки до пучка $0.15 \mathrm{~mm}$, толщина пучка $0.3 \mathrm{~mm}$, что соответствует нормированным параметрам: $G=5 \cdot 10^{-3}$, $B_{0}=0.1, B_{e}=0.2, \hat{\chi}=3.5$. При погонной мощности падающего излучения $100 \mathrm{~kW} / \mathrm{cm}$ (источником может служить субмиллиметровый гиротрон [16]), согласно рис. $8, a$, на нормированной длине пространства взаимодействия $L_{z}=4$ (что соответствует физической длине $11.6 \mathrm{~cm}$ ) может быть достигнут коэффициент усиления до $K=30 \mathrm{~dB}$. Мощность усиленного сигнала в этом случае составит $130 \mathrm{MW} / \mathrm{cm}$ при КПД 25\%.

\section{5. Заключение}

Заметим в заключение, что развитый подход может быть использован и при анализе мощных усилителей цилиндрической геометрии, поскольку при большой сверхразмерности структура симметричных ТМ-мод вблизи поверхности, вдоль которой движется трубчатый РЭП, может быть аппроксимирована соотношениями (4), (5). Важно также подчеркнуть, что квазиоптический подход легко обобщается на случай возбуждения ленточным электронным пучком поверхностных волн, распространяющихся над другими средами с отличной от (14) импедансной функцией. В частности, возможен анализ линейной и нелинейной стадии резистивной неустойчивости [17].

Работа выполнена при поддержке РФФИ, грант № 160200890 a. 


\section{Список литературы}

[1] Ковалев Н.Ф., Петелин М.И., Райзер М.Д. и др. // Письма в ЖЭТФ. 1973. Т. 18. Вып. 4. С. 32-36.

[2] Abubakirov E.B., Denisenko A.N., Fuchs M.I. et al. // IEEE Trans. on Plasma Sci. 2002. Vol. 30. N 3. P. 1041-1051.

[3] Коровин С.Д., Ростов В.В., Тотьменинов Е.М. // Письма в ЖТФ. 2005. Т. 31. Вып. 10. С. 17-25.

[4] Гришин Д.М., Губанов В.П., Коровин С.Д. // Письма в ЖТФ. 2002. Т. 28. Вып. 19. С. 24-31.

[5] Korovin S.D., Eltchaninov A.A., Rostov V.V. et al. // Phys. Rev. E. 2006. Vol. 74. N 1. P. 016501.

[6] Ельчанинов А.А., Коровин С.Д., Ростов В.В. и др. // Письма в ЖЭТФ. 2003. Т. 77. Вып. 6. С. 314-318.

[7] Ковалев Н.Ф., Петелин М.И., Райзер М.Д., Сморгонский A.B. // Релятивистская высокочастотная электроника / Под ред. А.В. Гапонова-Грехова. ИПФ АН СССР. 1979. C. $76-113$.

[8] Ковалев Н.Ф., Орлова И.М., Петелин М.И. // Изв. вузов. Радиофизика, 1968. Т. 11. Вып. 5. С. 783-786.

[9] Вайнштейн Л.А., Солнцев В.А. // Лекции по сверхвысокочастотной электронике. М.: Советское радио, 1973.

[10] Вайнштейн Л.А. // Электромагнитные волны. М.: Советское радио, 1957.

[11] Гинзбург Н.С., Заславский В.Ю., Малкин А.М., Сергеев А.С. // Письма в ЖТФ. 2011. Т. 37. Вып. 13. С. 31-39.

[12] Ginzburg N.S., Malkin A.M., Sergeev A.S., Zaslavsky V.Yu. // Appl. Phys. Lett. 2011. Vol. 99. P. 121505.

[13] Гинзбург Н.С., Малкин А.М., Железнов И.В. и др. // Письма в ЖТФ. 2013. Т. 39. Вып. 6. С. 50-59.

[14] Каценеленбаум Б.3. // Теория нерегулярных волноводов с медленно меняющимися параметрами. М.: Изд-во АН CCCP, 1961.

[15] Гинзбург Н.С., Зотова И.В., Муравьев А.А., Сергеев А.С. // ЖТФ. 2011. Т. 81. Вып. 3. С. 85-90.

[16] Glyavin M.Yu., Luchinin A.G., Golubiatnikov G.Yu. // Phys. Rev. Lett. 2008. Vol. 100. N 1. P. 015101.

[17] Гинзбург Н.С., Малкин А.М., Железнов И.В. и др. // Письма в ЖТФ. 2013. Т. 39. Вып. 2. С. 52-60. 